\title{
Qualitätsstrategie für das österreichische Gesundheitswesen
}

In zahlreichen gesetzlichen Grundlagen wird die Entwicklung einer gesamtösterreichischen Qualitätsstrategie gefordert. Das 2007 gegründete Bundesinstitut für Qualität im Gesundheitswesen hat von den politischen Entscheidungsträgern den Auftrag erhalten, diese Strategie gemeinsam mit den Stakeholdern zu entwickeln.

\section{Auszüge aus der Strategie \\ $\nabla$}

Die Qualitätsstrategie wird als Handlungsgrundlage verstanden, die regelmäßig auf ihre Gültigkeit, Anwendbarkeit und Umsetzung überprüft und entsprechend angepasst werden soll. Sie bestimmt den kontinuierlichen Verbesserungsprozess des österreichischen Gesundheitswesens.

Unter Gesundheitswesen verstehen wir die Krankenversorgung, in die der Hauptteil der verwendeten Ressourcen fließt, sowie die Gesundheitsförderung und Prävention, die an Bedeutung gewinnen. Die Qualitätsstrategie bezieht sich ausdrücklich auf alle diese Bereiche.

Unter Qualität wird der Grad der Erfüllung von patientenorientierter, transparenter, effektiver und effizienter Erbringung der Gesundheitsleistungen in allen Sektoren des Gesundheitswesens verstanden. Qualität ist das Maß der Übereinstimmung zwischen den Behandlungsergebnissen und den zuvor formulierten Zielen guter Behandlung.

Die Strategie richtet sich gleichermaßen an Entscheidungsträger und Gesundheitsdienstleister und trägt wesentlich zur flächendeckenden Sicherung und Verbesserung der Qualität in allen Sektoren des Gesundheitswesens bei. Sie unterstützt eine angemessene und insbesondere sichere Gesundheitsversorgung der Bevölkerung. Qualität wird zum Leit- und Steuerungskriterium des österreichischen Gesundheitswesens.

Im Sinne der Patientenorientierung sollen die jeweils betroffenen Menschen im Mittelpunkt der Entscheidungen und Handlungen stehen und befähigt werden, sich aktiv daran zu beteiligen. Die Sicherheit der Patientinnen und Patienten, der Mitarbeiterinnen und Mitarbeiter sowie des Systems stehen dabei im Vordergrund. Die Leistungen sollen effektiv im Sinne des bestmöglichen Ergebnisses und effizient im Sinne eines wirtschaftlichen Mitteleinsatzes erbracht werden.
Die Gleichbehandlung der Bürgerinnen und Bürger sowie Patientinnen und Patienten beim $\mathrm{Zu}-$ gang und der Inanspruchnahme von Gesundheitsdienstleistungen wird von allen Entscheidungsträgern und Gesundheitsdienstleistern unterstützt.

Ein partizipatives und kooperatives Vorgehen bei der Entwicklung und Umsetzung der Qualitätsstrategie sichert deren nachhaltige Wirkung. In dem Entscheidungen auf Grund rationaler, Evidenz-basierter Überlegungen getroffen werden, kann sich das Gesundheitswesen zu einem lernenden System entwickeln. Dabei sollen Anreize vor Sanktionen gesetzt und bei der Erbringung von Gesundheitsdienstleistungen ein berufsgruppenübergreifender und sektorenübergreifender Ansatz unterstützt werden. Größtmögliche Transparenz soll durch vergleichbare und valide Daten unterstützt und so die kontinuierliche Weiterentwicklung und Sicherung der Qualität im österreichischen Gesundheitswesen sichergestellt werden.

Autorenerklärung: Die Autorin erklärt, dass keine relevanten finanziellen Verbindungen in Bezug auf dieses Manuskript bestehen.
E.-M. Baumer

Qualitätsmanagement

Schlüsselwörter

Qualität

Gesundheitswesen

Österreich

OStrategie

Keywords

Oquality

health care

Austria

strategy
Institut

Gesundheit Österreich GmbH/ Bundesinstitut für Qualität im Gesundheitswesen, Wien, Österreich

Bibliografie

Dol $10.1055 / \mathrm{s}-0029-1242663$ Dtsch Med Wochenschr 2009; 134: S307 - (c) Georg Thieme Verlag KG Stuttgart - New York . ISSN 0012-0472

Korrespondenz

Eva-Maria Baumer, MPH

Gesundheit Österreich GmbH/ Bundesinstitut für Qualität im Gesundheitswesen

Stubenring 6

1010 Wien

Tel. +43151561-282

eMail eva.baumer@goeg.at 